\title{
MULTI-FREQUENCY STUDIES OF INTRINSIC INTRADAY VARIABILITY
}

\author{
STEFAN J. WAGNER \\ LSW Heidelberg, Germany
}

\begin{abstract}
Intraday variability is detected in Blazars throughout the electromagnetic spectrum. Only radio emission is affected by interstellar scintillation. The characteristics of variability observed at different frequencies are often similar and suggest that intrinsic variations also contribute to the changes detected in the radio wavelength regime. This in turn implies very high brightness temperatures.

Unambiguous signatures of scintillation and of intrinsic changes have been identified. The properties of intrinsic variations are reviewed to illustrate their potential contribution to variability in the radio domain. Such intrinsic changes need to be discriminated against when studying scintillation in quasars and BL Lac objects. Observations of scintillating sources and of sources with significant intrinsic IDV suggest interesting modifications to the standard paradigm of AGN.
\end{abstract}

\section{IDV - The Goal is Resolution}

Intraday Variability (IDV) has been known for many decades. Early reports of optical variations on short time-scales in Quasars and BL Lac objects have been reported by Oke (1967) and Harvey et al. (1972), respectively. Radio-observations of variations on time-scales shorter than one month have been reported during the 1970s already, and true IDV has been studied by Witzel et al. (1986) and Heeschen et al. (1987) with even faster variations being reported by De Bruyn (1988). Understanding its multifrequency aspects was ignored for a long time.

In the high-frequency regimes $\left(v>10^{11} \mathrm{~Hz}\right)$ it is straightforward to associate time-scales with light-travel diameters $(D=c \cdot \Delta t)$. This implies angular diameters of $\mu$-arcsec or less, and anywhere outside the radio domain this is the only way to probe the sub-milliarcsec structure.

At radio frequencies such derivations are affected by 'galactic seeing', i.e. interstellar scintillation, but irrespective of its cause IDV is the only way to probe the microarcsec regime: If the variations are intrinsic to the source, the concepts are similar to those applied at higher frequencies. If scintillation is important, one can constrain sizes using appropriate models and determine size-scales from the distance to the Fresnel zone. It is important to assess the different contributions and decompose intrinsic from scintillation effects wherever both are important on the same time scales. 
Intrinsic variations have interesting consequences for scintillation studies in different ways. Any source that is subject to intrinsic IDV is small enough to be subject to interstellar scintillation. In order to use scintillations as a tool to probe smallscale structure, it is necessary to rule out variability which is source-intrinsic, and any information from higher frequencies where scintillation is negligible, will help. On the other hand one might want to use scintillation to infer plasma properties, in which case sufficiently compact sources are needed and should be understood in order to serve as beacons.

\section{IDV in Blazars: All bands, All Time-Scales}

IDV sourees are flat-spectrum radio-sources, identified optically as FSRQs or BL Lac objects, now generally grouped together as Blazars. Blazar SEDs are very broad band, extending from $10^{8} \mathrm{~Hz}$ up to $10^{27} \mathrm{~Hz}$ in the most extreme cases. The lower frequency part is generally attributed to synchrotron emission, extending at least into the optical regime in all Blazars, but extreme cases have been found with synchrotron emission being observed to $10^{18} \mathrm{~Hz}$. At higher energies Compton-scattered radiation, either in the form of the synchrotron emission (SSC) or from external sources (dust, BLR, accretion disk; EC) plays an important role, and possibly dominates the high-energy SED. For an alternative view, see e.g. Mannheim, 1998. The spectral energy distributions of the sources are variable, indicating correlated variations at all energies.

IDV is most difficult to detect at the highest energies, where photon fluxes are very small. None of the Blazars detected by CGRO has been detected at a four sigma level in all the pointings on the specific object. The gamma-ray emission is only detected during flares, and even then average photon fluxes are of the order $10^{-7} \gamma \mathrm{sec}^{-1} \mathrm{~cm}^{-2}$, comparable to the detection of at most a few tens of photons per day. Significant variations of intensity on time-scales shorter than $10^{5} \mathrm{sec}$ can only be probed during the brightest flares. Whenever such bright states occur, IDV was detected (for a review see e.g. Hartman et al., 1997). BL Lac objects with high energy synchrotron cut-off frequencies have also been detected with ground-based Cerenkov telescopes at $\mathrm{TeV}$ energies. Variability studies in this energy range suffer from the same problems, i.e. an undetected (below threshold) DC component of the total flux, limiting studies to periods of flare-activity, and photon-noise limits for studies with high temporal resolution. IDV activity has clearly been detected by several groups (Aharonian, priv. comm., Catanese, priv. comm.). The shortest time scales detected at gamma-ray energies, require the emission sites to move with relativistic speeds. In environments of high photon density (as implied from the variability time scales), pair creation in interactions of gamma-rays with lower energy photons lead to effective attenuation of photons above the pair-creation threshold. Variability constrains the photon densities of target photons, and thus 
permits derivation of Doppler factors up to 8 in order to explain the observed $\mathrm{GeV}$ photon density (e.g. Mattox et al., 1997).

Rapid variations at $X$-ray energies have been discovered in most Blazars studied in detail. The $\mathrm{X}$-ray light curves are often very similar to those seen in optical or radio studies.

At optical frequencies IDV is detected in all Blazars. In many cases it has been possible to study variations on even shorter time-scales. Targeted searches for intrahour variability identified variations of up to 20 percent during a few minutes.

In the infrared regime IDV studies have been difficult. Observations have been limited to a few cases observed by IRAS and ISO. Sensitivity and sampling patterns have been insufficient to determine statistical properties, but significant variations on short time scales have been detected (e.g. Peng et al., 2000).

At radio frequencies, where IDV has been first discovered both the 'classical' IDV with time-scales on the order of $10^{5} \mathrm{sec}$ as well as faster variations (KedzioraChudczer, 1997; Dennet-Thorpe and De Bruyn, 2000 and this volume, and Jauncey, this volume) have been detected in 10 to $40 \%$ of flat-spectrum sources in different samples.

\section{Statistical Properties}

Blazars vary on a wide range of time scales. The characteristics of these changes can be probed over 4 orders of magnitude in the gamma- and X-ray domains and over up to 9 orders of magnitude at optical wavelengths. Since most of these studies involve very irregular sampling patterns, structure function analysis is a preferred means of a statistical description. They have been introduced in studies of radio-variability on short time scales by Heeschen et al. (1987) who distinguished between type 1 and type II sources of flickering, depending on the existence or absence of a pronounced shoulder in the structure functions on time-scales of about 20-30 hours. This break in the SF eventually lead to the term 'Intra-Day Variability'.

Optical studies of the same sources lead to structure functions with similar slopes and breaks ('shoulders') on the same time-scale $\left(10^{5} \mathrm{~s}\right)$ (Heidt and Wagner, 1996). Interestingly, X-ray bright BL Lac objects appear to have lower duty-cycles and amplitudes, but similar time-scales (Heidt and Wagner, 1998) despite significant differences in luminosity (the differences in redshift, which would result in differences in the apparent time-scales is too small to be detectable with the small samples studied so far). Recently X-ray monitoring over sufficiently long intervals and with proper sampling was carried out (Takahashi et al., in press, Kataoka et $a l$, in press). The structure functions again show very similar slopes and breaks on time scales of about $10^{5} \mathrm{sec}$. The similar location of the breaks, independent of photon-energy, indicaties that the temporal characteristics are not determined by 
the acceleration- or cooling times but rather by geometric quantities, such as the spatial extent of the accelerator.

IDV at high frequencies is unaffected by interstellar scattering. It is very broadband with similar characteristics over many orders of magnitude. There are close correlations observed over wide ranges in energy but light curves differ in detail. This is expected in view of the spectral changes observed throughout the NIR to $\mathrm{X}$-ray regimes.

\section{Intrinsie Radio-IDV}

The similarity of radio and optical variability suggests a common origin of the changes in flux density. Since optical variations are unaffected by scintillation, an intrinsic origin of radio-IDV is considered.* The main problems associated with radio-IDV are the concerns that flat-spectrum sources are supposed to be optically thick in the $\mathrm{GHz}$ regime, the apparent violation of the IC limit of 6 . $10^{11} \mathrm{~K}$ (Readhead, 1994), and the nearly symmetrical shapes of individual flares which suggest that radiative cooling times are comparable to acceleration and light-crossing times.

High-resolution imaging in the nearby-source M87 (where small angular scales probe small linear scales) have resolved structure down to $10^{17} \mathrm{~cm}$ (Junor et al., 1999). Although being less luminous than most IDV sources, this object also hosts a relativistic jet. In M87 the inclination against the line of sight is large, but it is obvious that the Doppler-boosted crossing times of the smallest resolved structures in this source are comparable to the variability time-scales of IDV sources if the jets were seen under small angles. The photosphere created by optical thickness appears to be much smaller than the scales probed by IDV - at least in this lower luminosity source.

The violation of the inverse-Compton limit seems to be severe. Apparent brightness temperatures of IDV in S5 0716+714, S4 0917+62, or S5 0954+654, are of order $10^{17}-10^{18} \mathrm{~K}$. Higher values have only been reported for sources 0405 385 (Kedziora-Chudczer et al., 1997, and these proceedings) and J1819+3845 (Dennet-Thorpe and de Bruyn, 2000). In the latter cases however, scintillation has been demonstrated to dominate the inter-hour variability (Jauncey, this volume,

* In principle, achromatic extrinsic variations, such as micro-lensing also need to be considered. As has been pointed out in Wagner (1992), Witzel and Wagner (1995), and in Wambsganss (this volume), micro-lensing-induced variability can be ruled out for most IDV sources for several reasons. The most important ones are: For a wide range of lens-masses, variability time scales are so short that micro-lensing would require relativistic motion in the source (i.e. intrinsic variations). Furthermore, the high duty cycle could only be provided in the regime of optically thick micro-lensing, which in turn would require an integral mass density of micro-lenses which is so high that macro-lensing is unavoidable. Since IDV objects are bright, flat-spectrum radio-sources, high S/N VLBI observations probe a sufficiently wide range in angular scales and dynamic range to rule out macro-lensing and thereby microlensing as a dominant contribution to IDV. 
Dennet-Thorpe, this volume). In $0716+714$ and $0954+654$ similarity in variability properties at optical and radio wavelengths suggest that scintillation is not the dominant process (Quirrenbach et al., 1991; Wagner et al., 1992; Wagner et al., 1996).

In the following we consider the hypothesis that the variability in some IDV sources is dominated by intrinsic variations and that photon densities can be directly determined from the variability time scales.

Apparent high brightness temperatures may be due to high Doppler beaming. Doppler factors up to 100 might be required in the most extreme cases. Note that the distribution of Doppler factors could not be obtained from the observed timescales, since most samples are severely biased by those sources which have the largest Doppler factors. An upper limit of $D=100$ was determined by Begelman et al. (1994), and the required values would be within physically acceptable limits. Such high Doppler factors have not been observed in VLBI experiments. Could this be due to an observational bias? Firstly, the sampling pattern of VSOP or mmVLBI experiments is strongly biased against detecting ultra-fast components. IDV sources have high duty cycles and rather low amplitudes. If individual flares could be identified with blobs of enhanced density in the same way as 'classical' VLBI knots are associated with major (and longer lasting) outbursts, the velocities of such structures would be high. On the other hand, even if they could be identified in maps of limited dynamic range, fast-moving knots would easily be confused in experiments of the sampling rate obtained in normal VLBI experiments. Furthermore it is a well-known problem that the apparent velocities determined in VLBI experiments might not be representative of the flow velocity, which could actually be much higher. If the low amplitudes are indicative of low contrast, the fluid elements responsible for IDV would be identified with the smooth flow rather than the (potentially slower or even steady) shocks which are identified with VLBI features.

Another bias could be caused by the angular resolution that can be achieved. Decelerating jets would be much faster at smaller radii which cannot yet be explored. Compton drag on compact $(<50 \mu$ arcsec $)$, ultra-fast jets might contribute to the matter-loading of jets close to the sites of jet-acceleration, contribute to the huge radiative losses in the high-energy end of the spectrum and slow down intrinsically fast jets to the velocities detected in VLBI experiments. Such a possibility is in line with the systematically higher velocities found by Marscher et al. (2000) in high-frequency VLBI experiments.

High velocities are not the only possible explanation for the high apparent temperatures. Doppler factors may be significantly relaxed in certain geometrical configurations. In addition to the models discussed in Qian (1991), Marscher (1992), and Wagner and Witzel (1995). Spada et al. (1999) recently suggested oblique shocks in conical jets as an interesting way to obtain $T_{B}$ up to $3 \cdot 10^{17} \mathrm{~K}$.

It would also be possible that the brightness temperatures are indeed higher then several $10^{11} \mathrm{~K}$. The inverse Compton (IC) limit of $6 \cdot 10^{11} \mathrm{~K}$ for incoherent electron 
synchrotron emission could be violated for short periods of time. The limit might also be shifted to higher values. It has been speculated that proton synchrotron emission or coherent synchrotron radiation might be at work, both of which would increase the brightness temperature at which catastrophic Compton cooling would set in.

Many IDV sources are likely to move relativistically with high Doppler factors (as derived from direct VLBI observations or inferred from the rapid variability detected in the gamma-ray range). A Doppler correction of the apparent brightness by at least $D^{3} \sim 1000$ is unavoidable. It is interesting to note that the extreme cases of scintillation in 1819+3845 and PKS 0405-385 imply intrinsic brightness temperatures above the IC limit. For a screen at $500 \mathrm{pc}$ distance, Kedziora-Chudczer $e t$ al. determine $T_{b}^{\text {intr }} \sim 5 \times 10^{14} \mathrm{~K}$. This can be reconciled with the IC by assuming a Doppler factor of 1000, which would be much higher than those proposed for intrinsic IDV or it may support radiation mechanisms with higher limiting brightness temperatures.

It appears that both IDV due to scintillation and intrinsic variations suggest very high intrinsic brightness temperatures. If the intrinsic limit was of order $10^{13}-$ $10^{14} \mathrm{~K}$, directly observed Doppler factors of order 20 would be sufficient to explain apparent temperatures of $10^{17}-10^{18} \mathrm{~K}$.

\section{Acknowledgements}

I would like to thank Arno Witzel for many years of fruitful collaboration. Two anonymous referees helped improving the text. The DFG provided funds for attending this conference (WA 720/12-1) and had supported IDV research through SFB 328.

\section{References}

Begelman, M., Rees, M. and Sikora, M.: 1994, Astrophys, J. Lett. 429, 57-60.

de Bruyn, A.G.: 1988, Proceedings of IAU Symp. 129, 97-98.

Dennet-Thorpe, J. and de Bruyn, A.G.: 2000, Astrophys. J. Lett. 529, 65-68.

Hartman, R.C., Collmar, W., von Montigny, C. and Dermer, C.D.: 1997, Proceedings Fourth Compton Symposium, AIP $\mathbf{4 1 0}$ (1), 307-328.

Harvey G.A., et al.: 1972, Astrophys. Lett. 11, 147-492.

Heidt, J. and Wagner, S.J.: 1996, Astron. Astrophys. 305, 42-52.

Heidt, J. and Wagner, S.J.: 1998, Astron. Astrophys. 329, 853-862.

Heeschen, D., Krichbaum, T., Schalinski, C. and Witzel, A.: 1987, Astron. J. 94, 1493-5072.

Junor, W., Biretta, J.A. and Livio, M.: 1999, Nature 401, 891-892.

Kedziora-Chudczer, L., Jauncey, D.L., et al.: 1997, Astrophys. J. Lett. 490, 9-12.

Mannheim, K.: 1998, Science 279, 684-686.

Marscher, A., Marchenko-Jorstad, S., Mattox, J., Wehrle, A. and Aller, M.: 2000, Proceedings: Astrophysical Phenomena Revealed by Space VLBI, ISAS, 39-46. 
Maxschcr, A.P.: 1992, Proceedings: Physics of Active Galactic Nuclei, Springer, p. 510. Mattox, J.R., Wagner, S.J., Malkan, M., et al.: 1997, Astrophys. J. 476, 692-712.

Oke, J.B.: 1967, Astrophys. J. 147, 901-907.

Peng, B., Krans, A., Krichbaum, T.P., Müller, S., et al.: 2000, Astron. Astrophys. 353, 937-943.

Qian, S.-J., Quirrenbach, A., Witzel, A., et al.: 1991, Astron. Astrophys. 241, 15-21.

Quirrenbach, A., Witzel, A., Wagner, S.J., et al.: 1991, Astrophys. J. 372, L71-L74.

Readhead, A.: 1994, Astrophys. J. 426, 51-59.

Spada, M., Salvati, M. and Pacini, F.: 1999, Astrophys. J. 511, 136-141.

Wagner, S.J.: 1992, Proceedings: Gravitational Lenses, Springer, 189-195.

Wagner, S.J. and Witzel, A.: 1995, Annu. Rev. Astron. Astrophys. 33, 163-197.

Wagner, S.J., Witzel, A., Krichbaum, T.P., et al.: Astron. Astrophys. 271, 344-352.

Wagner, S.J., Witzel, A., Heidt, J., et al.: Astron. J. 111, 2187-2212.

Witzel, A., Heeschen, D., Schalinski, C., et al.: 1986, Mitt. Astron. Ges. 65, 239-241. 